Página inicial: 447 - Página final: 455

TIPO DE ARTÍCULO: de Investigación

\title{
REVISIÓN A LA IMPLEMENTACIÓN DE LOS PROGRAMAS DE RETORNO. UNA MIRADA A PARTIR DE TRES ESTUDIOS DE CASO EN EL ORIENTE ANTIOQUEÑO.
}

\author{
REVISION TO THE IMPLEMENTATION OF RETURN PROGRAMS. \\ A LOOK INTO THREE CASE STUDIES IN EASTERN ANTIOQUIA.
}

\author{
RECIBIDO: ENERO 2015 REVISADO: ABRIL 2015 ACEPTADO: 2 DE MAYO DE 2015
}

Por: Luis Antonio Ramirez Zuluaga. ${ }^{1^{*}}$

\section{RESUMEN:}

El presente artículo es producto de la investigación "Análisis de los procesos de retorno y reubicación de población en situación de desplazamiento forzado en los municipios de la subregión del oriente de Antioquia: tres estudios de caso", inscrita en la Facultad de Derecho de la Universidad Cooperativa de Colombia, sede Medellín. Los estudios de caso se adelantan en los corregimientos El Jordán (municipio de San Carlos), Santa Ana (municipio de Granada) y Aquitania (municipio de San Francisco). La investigación se centra en analizar la efectividad de los procesos y politicas de retorno que se implementan en los lugares en cuestión. Para ello se tiene en cuenta el marco normativo que debe aplicarse a dichos procesos, así como los protocolos tendientes a darles una ruta metodológica. En el transcurso de la investigación, se accede además a información directa brindada por las comunidades retornadas y los entes administrativos locales encargados de los procesos de retorno. A partir del análisis de la información brindada por estos actores se concluye que la efectividad de los programas de retorno presenta diversas falencias respecto al goce efectivo de los derechos de las personas que han sido víctimas de desplazamiento forzado.

\section{PALABRAS CLAVE:}

Desplazamiento forzado; Procesos de retorno; Políticas públicas; Dignidad; Goce efectivo de derechos.

\begin{abstract}
:
This article was the product of the research "Analysis of the processes of return and relocation of the population in a situation of forced displacement in the municipalities of the sub-region of Eastern Antioquia: three case studies," registered in the Faculty of Law at Cooperative University of Colombia, Medellin campus. The case studies were carried out in the villages of El Jordan (Municipality of San Carlos), Santa Ana (Municipality of Granada), and Aquitaine (Municipality of San Francisco). The research focused on analyzing the effectiveness of the processes and policies of return, which are implemented in the places in question. To that end, the regulatory framework to be applied to these processes, as well as the protocols aimed at giving them a methodological path is taken into consideration. In the course of this research, direct information is provided by returnee communities and local administrative bodies responsible for return processes. Based on an analysis of information provided by these actors, it is concluded that the effectiveness of return programs presents several shortcomings with respect to the effective enjoyment of the rights of people who have been victims of forced displacement.
\end{abstract}

\section{KEY WORDS:}

Forced displacement, return processes, public policies, dignity, and effective enjoyment of rights.

\footnotetext{
$1^{1^{*}}$ Magister en Filosofia de la Universidad Michel de Montaigne, Burdeos-Francia; actualmente cursa el Doctorado en Filosofia de la Universidad de Antioquia. Profesor - Investigador de la Facultad de Derecho de la Universidad Cooperativa de Colombia, sede Medellín, Colombia. Contacto: Luis.ramirezme@campusucc.edu.co
} 


\section{Introducción.}

Una de los problemas sociales que con mayor fuerza se ha sentido en las últimas décadas en Colombia, como expresión del conflicto y la violencia armada padecida en gran parte del territorio nacional, ha sido la del desplazamiento forzado.

Hasta mayo de 2011 la Agencia Presidencial para la Acción Social y la Cooperación Internacional (Acción Social) registraban más de 3,7 millones de desplazados internos en el país. Entretanto la Consultoría para los Derechos Humanos y el Desplazamiento Forzado (CODHES) ha considerado que la cifra real de desplazados por el conflicto armado interno desde mediados de los años 80 supera los 5 millones de personas.

Históricamente Antioquia ha sido uno de los departamentos del país con mayores niveles de expulsión violenta, asî como de recepción de población víctima del desplazamiento forzado. Debido a múltiples causas, factores e intereses, entre ellos, las disputas entre los actores del conflicto armado interno y las agresiones contra la población civil, se produjeron una gran cantidad de expulsiones forzadas en la subregión del oriente antioqueño, llevando a que una parte significativa de los municipios que la componen vieran disminuida su población de manera vertiginosa, incluso por encima de la mitad de la misma.

En los casos específicos de zonas tan alejadas como el corregimiento El Jordán del municipio de San Carlos, el corregimiento Santa Ana del municipio de Granada y el corregimiento de Aquitania del municipio de San Francisco, hubo ciertamente a comienzos de la primera década del presente siglo un desplazamiento forzado de más de la mitad de la población; incluso algunas veredas o zonas rurales de estos corregimientos quedaron completamente despobladas a causa del desplazamiento. En los cascos urbanos de Santa Ana y Aquitania esta problemática llegó a ser tan aguda que entre el 2003 y el 2004 se convirtieron en pueblos fantasmas, quedando tan solo tres o cinco familias.

Como una de las alternativas para la superación definitiva del problema del desplazamiento forzado, los marcos jurídicos y de política pública del Estado colombiano han previsto el desarrollo de procesos de retornos y reubicaciones, con la observancia de principios y estándares internacionales, tales como la voluntariedad, la seguridad y la dignidad. Pese a que estas estrategias se vienen desarrollando desde hace varios años, y que se encuentran establecidos en la política pública nacional parámetros para diseñar una metodología que permita realizar un acompañamiento técnico de los retornos y las reubicaciones, se presenta un gran vacío en cuanto a la revisión exhaustiva de estos procesos que permita valorar el impacto que ellos han generado en términos del goce efectivo de derechos establecidos en la Sentencia T 025 de 2004. En este orden de ideas, hoy en día se hace necesario identificar los factores jurídico-políticos que permitan evidenciar el verdadero impacto de los procesos mencionados.

\section{Metodología.}

Se han realizado tres estudios de caso sobre los diferentes procesos de retornos y reubicaciones seleccionados en la subregión del oriente de Antioquia, en los cuales se han podido contrastar las medidas y acciones realizadas por las autoridades públicas con relación al cumplimiento de los principios y estándares establecidos para dichos procesos. La recolección de información se ha hecho a partir de tres técnicas: encuestas, grupos focales y entrevistas. A las comunidades retornadas se les ha aplicado las tres técnicas y a las personas responsables de los entes administrativos encargados de los procesos de retorno (Personerías, Alcaldias y Unidades de Atención a Víctimas) sólo se les ha hecho entrevista. 


\section{Resultados.}

Debido a la disminución sustancial de las acciones bélicas en el marco del conflicto armado, varias entidades públicas del orden nacional, departamental y municipal, desde el año 2004, comenzaron procesos de retorno y reubicación en los corregimientos y municipios de la subregión del oriente que se han mencionado. Estos procesos se han desarrollado en el marco de políticas del orden nacional como "Retornar es Vivir" y "Familias en su Tierra". Igualmente, se han presentado alianzas entre la alcaldía de Medellín con algunos municipios de la subregión del oriente, entre las cuales se destacan las desarrolladas con los municipios de San Carlos y Granada.

Desde el corregimiento de Santa Ana, en octubre de 2009, la política pública denominada "Retornar es vivir" fue mediatizada y publicitada por entes gubernamentales de esa época como el Ministro de Defensa, el Director de la entonces llamada Acción Social y el Gobernador de Antioquia. Esa publicidad y el impacto que se venía generando con la "Alianza Medellín San Carlos", crearon un boom de los retornos que hizo que la atención de la opinión pública se dirigiera a los municipios de Granada y San Carlos para ser reconocidos bajo el apelativo de "cuna del retorno del oriente antioqueño". Tal boom condujo a que en los últimos seis años se haya dado un retorno masivo a dichas localidades.

Sin embargo, ese retorno masivo ha ocurrido, en su mayor parte, de manera individual y sin acompañamiento institucional desde la fase exploratoria de los procesos de retorno (Agencia presidencial para la acción social y la cooperación internacional, 2006, p 8) ; (Unidad para la Atención y Reparación Integral a las Víctimas UARIV, 2014 p 77-78), fase en la cual se deben verificar los principios de voluntariedad, dignidad y seguridad que son los fundamentos propuestos por las Naciones Unidas (1998) para restablecer los derechos a quienes han sido víctimas de los desplazamientos internos. En Colombia, como garantías de los procesos de retorno, desde la Ley 387 de 1997 se asumieron unos criterios similares a los de los principios referidos, añadiendo el principio de estabilización socio-económica; en concordancia con esa norma promulgada en 1997, también la Ley 1448 de 2011 -Ley de Víctimas y Restitución de Tierras- ha reconocido esos mismos principios para la activación de las medidas de reparación para con las víctimas del desplazamiento forzado.

En aplicación de lo estipulado por dichas leyes, las políticas públicas "Retornar es vivir" y "Familias en su tierra" han contado ya con dos protocolos, uno de corte básico emitido en el 2006 durante la operación de la ya desaparecida Acción Social y otro más elaborado que fue publicado en el 2014 por la actual Unidad para la Atención y Reparación Integral a las Víctimas (UARIV). Los protocolos que pretenden dar unos fundamentos metodológicos a los procesos de retorno se han venido pues haciendo sobre la marcha; además han derivado no sólo en el aumento de entidades y funcionarios encargados de atender a la población retornada -lo cual parece necesario dada la magnitud del problema del desplazamiento que le es correlativo a los retornos-, sino que también han incrementado los trámites y el tiempo para que se dé un retorno efectivo.

El masivo regreso individual, y sin acompañamiento, que trajo el boom de los retornos en las localidades del oriente antioqueño ha tenido además dos elementos conexos. Uno está relacionado con lo mencionado al final del párrafo anterior y consiste en que el tiempo que se debe esperar para obtener las garantias institucionales del retorno es demasiado largo; una vez se solicita el inicio del proceso, pueden pasar entre cuatro y seis meses para que desde la unidad de víctimas se empiecen a verificar los principios de voluntariedad, seguridad y dignidad. Ante el desespero o las penurias de las personas demandantes, muchas prefieren entonces regresar sin el acompañamiento institucional. El otro elemento tiene que ver con 
el hecho de que mucha gente desconoce el procedimiento a seguir y simplemente regresan sin acompañamiento.

En los municipios que reciben las personas que regresan, son las personerias y las oficinas de atención a víctimas las que se ven desbordadas por las demandas de quienes vuelven sin seguir el protocolo para los procesos de retorno. Es tal la dimensión del problema que se ha generado con el número de "desplazados retornados" a los municipios de Granada, San Carlos y San Francisco, que en los tres se ha declarado la "emergencia por retornos".

A este desbordamiento de la problemática en cuestión, se le suma el hecho de que han sido insuficientes los cupos generados desde las diferentes fases de la política "Familias en su tierra" con la cual se le ha dado continuidad en los últimos años a "Retornar es vivir".

En una entrevista concedida por el personero de Granada en octubre de 2014, este manifestó que "desde lo local se hace lo que toca y lo que se puede hacer"; y es que, en general, los funcionarios locales se encuentran excedidos por la cantidad de declaraciones, casos y procedimientos que deben atender con el regreso masivo de desplazados.

Con respecto al número de familias y de personas que han retornado existen evidentes incongruencias entre las cifras que se han manejado desde entes estatales y aquellas que tienen los mismos municipios; incluso estos últimos se ven a veces contrariados entre la información que les llega desde el Departamento de Prosperidad Social y lo que ellos van recibiendo y tratando de agenciar día a día.

Según la Asociación de Campesinos de Antioquia (2011), en la época en que operaba Acción Social se publicitaban cifras de retornados mayores a la población de localidades como las de Granada y San Francisco. Es dificil de establecer el consolidado de datos que pueda tener hoy en día el Departamento de Prosperidad Social (DPS) a través de la Unidad de Atención a Víctimas, ya que se manejan cifras parciales de acuerdo a cada una de las fases que lleva "Familias en su tierra" y según lo han manifestado los funcionarios de las personerías y de las oficinas locales de Atención a Víctimas de los municipios de Granada y San Carlos, hay nombres de personas que se repiten entre una y otra fase de esa política pública; además, es posible que en el DPS exista un subregistro de personas retornadas ya que muchas lo han hecho sin seguir los conductos estipulados para los procesos de retorno que se manejan desde el Estado. En febrero de 2015 se envió un derecho de petición a la Unidad de Atención y Reparación Integral a las Víctimas (oficina territorial de Antioquia), solicitando los datos de las personas desplazadas y retornadas de los corregimientos de Aquitania, Santa Ana y El Jordán, pero a la fecha no han dado respuesta.

Ello ha conllevado a un problema en la sistematización de la información tanto de las personas que retornan como en el seguimiento de todo el proceso. Un seguimiento que ha brillado por su falta de planeación y que para el caso, por ejemplo, de los proyectos productivos ofertados a quienes han retornado se evidencia que no siempre existe un acompañamiento técnico; además, los acompañamientos técnicos (llevados por operadores como la OIM o la Corporación Ayuda Humanitaria) no han sido rigurosos ni mantenidos en el tiempo.

Si se toma la asistencia en proyectos productivos como componente de "la estabilización socio-económica", es entonces dificil llegar a la conclusión de que ésta se dé efectivamente en la mayor parte de los casos; sólo muy pocas personas han logrado desarrollar proyectos productivos "exitosos". 
En cuanto al acompañamiento para la comercialización de productos, también se evidencian problemas por la falta de garantía de un precio justo y digno.

Con respecto a un seguimiento o acompañamiento que garantice la seguridad y/o soberanía alimentaria de las comunidades, también se hallan falencias, pues hay quienes dejan de cultivar o no se ocupan lo suficiente de sus cultivos, prefiriendo esperar las ayudas económicas del Estado y dejando a un lado la tradición campesina.

En términos generales, puede decirse que el acompañamiento estatal ha sido insuficiente y que desde el Estado se ha tomado como medida más general de la reparación el otorgar dinero:

La indemnización como único y último modo de reparación, una indemnización a la que tampoco se le hace seguimiento para valorar la óptima inversión de los recursos recibidos. Es más, lo que sería el supuesto "proceso de retorno" se asume que se finaliza cuando se hace la entrega de la reparación económica, pero lo que se evidencia es que existen tantos vacíos en el "proceso de retorno" que difícilmente se puede hablar de "proceso".

Lo que se ha configurado desde el Estado y sus políticas públicas es una imposición de metodologias descontextualizadas y desarticuladas, que desconocen avances y/o propuestas generadas desde lo local; son estas últimas las que se construyen de una manera más coherente o de mayor conformidad con las necesidades identificadas en los territorios o localidades. Dentro de todo el andamiaje burocrático del Estado, emergen funcionarios locales muy comprometidos que resultan ser una especie de paliativo de las decretadas emergencias por retorno. Aunque la política pública fue planeada desde el orden nacional sin consenso con los territorios, existen en los territorios funcionarios prestos a modular dichas politicas en aras de superar deficiencias con respecto a la atención de las comunidades con las que están comprometidas.

La falta de información de la Unidad para la Atención y Reparación Integral a las Víctimas UARIV (Antioquia) y las incongruencias entre los datos de la escala nacional y local, hace dificil presentar unos datos precisos acerca del número de personas retornadas; sin embargo, gracias a las entrevistas que se han tenido con pobladores y entes administrativos de los municipios objeto de análisis de este estudio, lo que se ha podido detectar en el transcurso de nuestra investigación con respecto a los procesos de retorno es una información preliminar y cualitativa que se expone a continuación:

Pese a que al parecer en El Jordán sólo ha retornado menos del 50\% de la población desplazada, entre los tres corregimientos analizados es éste donde los procesos de retorno se han dado de un modo más óptimo. Ello se explica, por un lado, porque dicho corregimiento también fue favorecido por la "Alianza Medellín-San Carlos" a través de la cual fue ejecutado un "retorno colectivo" con un verdadero acompañamiento inicial; y por otro lado, porque algunas de las personas que han retornado han tenido, además de los proyectos productivos brindados dentro de las políticas públicas, algunas posibilidades de trabajo como las que ofrece la central hidroeléctrica de San Carlos que opera en la zona y que en el 2014 generó empleo para 245 personas del área urbana y rural de dicho corregimiento.

Los pobladores de Aquitania dicen que ha retornado aproximadamente el $50 \%$ de la población desplazada. Sin embargo, lo han hecho en su mayoría sin acompañamiento. Tanto en el casco urbano como en el rural son muy visibles aún las huellas del conflicto y hasta el momento son pocos los proyectos productivos que han tenido éxito debido a que la comercialización de productos resulta muy laboriosa, pues su infraestructura vial se encuentra en condiciones deficientes. Los pobladores demandan incluso como "reparación 
colectiva" que se les pavimente la carretera que del corregimiento conduce a la autopista Medellín-Bogotá.

En Santa Ana se tiene aún también una muy deficiente infraestructura vial; los 24 kilómetros que la separan de Granada se recorren en dos horas y media. Alli ha retornado menos del 50\% de la población desplazada: 192 familias según lo advierte la oficina de atención a víctimas de Granada. Entre las personas retornadas, muchas residen en el casco urbano del corregimiento o fluctúan entre éste y el municipio de Granada. En la zona rural de Santa Ana hay aún veredas con minas antipersona, lo cual ha dificultado el regreso de personas que están a la espera de iniciar un proceso de retorno.

\section{Conclusiones.}

En primer lugar, se evidencia la desarticulación entre las instituciones del aparato Estatal y de diversa indole territorial: nacional, regional y local. Por un lado, los programas que se lideran desde la Unidad de Atención y Reparación Integral a Víctimas -UARIV- son operados y ejecutados por contratistas que, de acuerdo a los intereses y voluntades politicas de quienes asignan los contratos, entran hacer parte del engranaje burocrático y por lo cual no siempre hay continuidad de dichos operadores ni de los profesionales que acompañan los planes en una intermitencia de empresas y personas que en medio de los trámites y tiempos que lleva suscribir contratos con entidades del Estado, retardan la atención efectiva y oportuna.

Por otro lado, a lo largo del tiempo en que se ha llevado a cabo los programas para atención de las víctimas, los trámites y procedimientos de las políticas y gobiernos de turno se han construido y reconstruido, no sólo sobre la marcha, sino también sin la adecuada coordinación institucional y rigurosidad que permitan tener un conocimiento claro y una información consolidada y sistematizada de la población desplazada; se deduce entonces un gran subregistro de la población que se encuentra en esta condición.

Sumado a los formatos y trámites que deben surtir las personas para obtener atención y acompañamiento, existe también desconocimiento y poco acceso a la información de cómo se debe proceder en estos casos, hecho que se evidencia con el boom del retorno que se presentó en los municipios objeto de este estudio y que puso de manifiesto la incapacidad institucional para atender a la población.

En segundo lugar, hay un desconocimiento de las dinámicas y contextos locales, así como de los cambios y transformaciones sufridas por las familias desplazadas que conllevan nuevas realidades y nuevos intereses; lo cual ha derivado en que los proyectos y programas implementados para "asegurar" las condiciones mínimas de las familias retornadas -es decir, la "estabilización socio económica"-, tiendan al fracaso.

Los protocolos producidos e impuestos desde el orden nacional han desencadenado el surgimiento de escenarios adversos que dificultan que se den verdaderos procesos para asegurar la no vulneración de derechos y el goce efectivo de los mismos de la población víctima de desplazamiento.

Así púes, como ya se mencionó, la intermitencia de las empresas operadoras de los programas, así como sus funcionarios, muchos de los cuales son de otras zonas del país, resultan ajenos a las realidades locales, por lo cual el acompañamiento es fragmentario e insuficiente. 
En lo que respecta al componente productivo de la estabilización socio-económica se tiene, por un lado, no sólo poco acompañamiento sino también que, dada la diversidad de los proyectos productivos, la asistencia técnica muchas veces no corresponde necesariamente a la experticia de los profesionales a cargo; adicionalmente, no se cuenta ni con el tiempo ni con los recursos para el establecimiento real de los proyectos ni el encadenamiento productivo y comercial de los productos.

Por otro lado, subsiste una realidad que no puede desconocerse y es la pérdida de la vocación agrícola de la población desplazada, pues los largos periodos del desplazamiento generaron que algunas familias se vieran obligadas a aprender y desarrollar otros medios para su subsistencia; así mismo, muchos de los hijos de los campesinos eran sólo niños en el momento del desplazamiento y retornaron siendo jóvenes y adultos sin ningún arraigo o interés por el campo y con el agravante quizá de que llevan consigo los patrones culturales y costumbres propios de un contexto citadino.

En cuanto al acompañamiento psicosocial, nuevamente la intermitencia de los operadores y profesionales a cargo de este componente, así como la limitación de tiempo y recursos que están supeditados a los plazos contractuales y a la entrega de productos, desbordan y no permiten que la atención psicosocial sea efectiva, pues ésta se ha limitado al trabajo con grupos, marginando la atención individual y dejando sin acceso a muchas personas ya que están dirigidos al cumplimiento de ciertos requisitos (edad, sexo, etc.) y no al proceso en sí que se requiere para atender fenómenos tan complejos como las heridas que deja la guerra.

En consecuencia, las medidas implementadas en los componentes productivos y social para la estabilización socio-económica de la población en proceso de retorno resultan siendo medidas cortoplacistas donde se reduce la reparación a presentar cifras y números de personas participantes de uno u otro programa y cantidad de dinero invertido o entregado a las familias, dejando a la población aún en condiciones de vulnerabilidad económica y social; primero, porque no cuentan con estabilidad económica que garantice la subsistencia en condiciones dignas; y segundo, porque este tipo de acompañamiento psicosocial tan fragmentario resulta más dañino en tanto aún persisten traumas, heridas abiertas y dolores que no cesan y no permiten la reconstrucción del tejido familiar y comunitario.

Además, este tipo de medidas conllevan un gran riesgo cuando la reparación se reduce a las cifras y al pago de indemnizaciones, pues como víctimas del desplazamiento y ante la imposibilidad de llevar una vida digna es mucho más fácil, y con menos esfuerzo para algunas familias, vivir del asistencialismo Estatal.

No obstante, el acercamiento a los contextos y dinámicas de los corregimientos de El Jordán, Aquitania y Santa Ana y los datos obtenidos durante el trabajo de campo, permiten evidenciar procesos sociales gestados desde lo local que dan cuenta de la potencialidad de las capacidades de los actores locales para sobreponerse y hacerle frente, no sólo al desplazamiento, sino también a todos los hechos victimizantes.

Ante las dificultades mencionadas anteriormente para la implementación de la atención integral a las víctimas de desplazamiento desde el orden nacional, aparecen funcionarios vinculados a diferentes secretarías de despacho de los municipios, las personerias municipales y otras instancias del orden local que desde su rol han apoyado fuertemente los procesos de retorno en sus municipios; muchos de ellos, también víctimas y originarios de la zona, tienen sentido de pertenencia, conocimiento del contexto y un sentido de solidaridad con la población objeto de atención que de alguna manera subsana, no como se quisiera, las falencias del programa de atención a víctimas. 
A partir de los tres estudios de caso analizados, los cuales ponen en entredicho la efectividad de la política nacional para la atención a la población desplazada y sus consiguientes planes de retorno, encontramos una luz que podría orientar el camino para la atención real y efectiva de las víctimas del desplazamiento y que emana de procesos sociales gestados desde lo local.

En los estudios de caso se encontraron iniciativas locales, muchas de ellas emanadas de la sociedad civil, como: La Legión del Afecto que desde el municipio de San Luis brindó apoyo a la población retornada del corregimiento de Aquitania en San Francisco; ASOVIDA y el Salón del Nunca Más en Granada y el Centro de Acercamiento para la Reconciliación y Reparación CARE del municipio de San Carlos. Desde diferentes escenarios, la sociedad civil se ha organizado buscando alternativas y mecanismos para trabajar en pro de la población y generarle mejores condiciones que van mucho más allá de lo económico y tienden a fortalecer el tejido social.

También se encontraron estrategias de los gobiernos locales como las mesas de salud mental en los municipios de San Carlos y Granada, y aunque su permanencia puede estar supeditada a los gobiernos de turno, se está intentando desde una iniciativa de los gobiernos locales brindar atención.

Así mismo, la población joven, principalmente de los municipios de San Carlos y Granada, se encuentra empoderada desde las casas de la cultura, institutos de deporte y redes municipales de juventud y vienen liderando proyectos culturales, artísticos y deportivos, tales como los juegos por la paz y la integración del oriente antioqueño y la estrategia de Animarte, por dar algunos ejemplos, como mecanismos transformadores de la realidad que aportan a la reconstrucción del tejido social mediante la generación de espacios para el encuentro comunitario. A partir de estas experiencias, podríamos decir que el principio que debiera operar para buscar soluciones al proceso de retorno es la construcción conjunta de alternativas a los problemas desde abajo, en reconocimiento, en principio, de las expectativas e intereses de las comunidades y de sus potencialidades para el cambio y la transformación social, y no desde afuera, que aunque con buenas intenciones, éstas se desdibujan en su aplicación.

En este sentido, las políticas y planes deberian estar orientados a brindar herramientas para el empoderamiento de las familias y comunidades objeto de retorno y trabajar conjuntamente en la construcción de planes de vida y la reconstrucción del tejido social, atendiendo a las particularidades y potencialidades de cada territorio y su población, permitiéndoles así trascender la condición de víctimas y convirtiéndose en ciudadanos(as) partícipes y/o activos(as) que finalmente puedan obtener el goce efectivo de sus derechos. 


\section{Referencias Bibliográficas.}

Agencia presidencial para la acción social y la cooperación internacional. (2006). Protocolo para el acompañamiento a los procesos de retorno o reubicación de población desplazada. Bogotá: Acción social-Presidencia República de Colombia.

Asociación de Campesinos de Antioquia. (2011). Noticias del Oriente Antioqueño. Recuperado el Mayo de 2014, de La inconsistencia de las cifras oficiales sobres los Procesos de Retorno en San Francisco: https://noticiasorienteantioqueno.wordpress.com/2011/12/19/1ainconcistencia-de-las-cifras-oficiales-sobres-los-procesos-de-retorno-en-san-francisco/

Balvín Alvarez, J. W., Insuasty Rodriguez , A., \& Cadavid Acevedo, P. (2009). Victimas, Violencia y despojo. Medellín: Litoimpacto.

Corte Constitucional. (2004). Sentencia T-025. Obtenido de corteconstitucional.gov.co : http://www.corteconstitucional.gov.co/relatoria/2004/t-025-04.htm

Naciones Unidas. (1998). Principios Rectores de Desplazamientos Internos. ACNUR.

OCHA. (2002). Guia para la aplicación de los principios rectores de los desplazamientos internos. Bogotá: Codice ltda.

República de Colombia. (1997). LEY 387. "Por medio de la cual se adoptan medidas para la prevención del desplazamiento forzado; la atención, protección, consolidación y estabilización socio-económica de los desplazados internos por la violencia en la República de Colombia".

República de Colombia. (2011). LEY 1448. "Por la cual se dictan medidas de atención, asistencia y reparación integral a las víctimas del conflicto armado interno y se dictan otras disposiciones".

Unidad para la Atención y Reparación Integral a las Víctimas UARIV. (2014). Protocolo para el acompañamiento a retornos y reubicaciones en el marco de la reparación integral a víctimas del desplazamiento forzado. Républica de Colombia. 
